\title{
Horizons/Théâtre
}

Revue d'études théâtrales

8-9 | 2016

Théâtres du geste, du jeu et de la voix

\section{À quoi jouons-nous ? Jouer, rejouer, déjouer la scène contemporaine}

\section{Aline Jaulin}

\section{(2) OpenEdition}

1 Journals

Édition électronique

URL : https://journals.openedition.org/ht/777

DOI : $10.4000 /$ ht.777

ISSN : 2678-5420

Éditeur

Presses universitaires de Bordeaux

\section{Édition imprimée}

Date de publication : 31 décembre 2016

Pagination : 54-66

ISSN : 2261-4591

\section{Référence électronique}

Aline Jaulin, «À quoi jouons-nous ? Jouer, rejouer, déjouer la scène contemporaine », Horizons/Théâtre [En ligne], 8-9 | 2016, mis en ligne le 01 janvier 2018, consulté le 18 mai 2022. URL : http:// journals.openedition.org/ht/777 ; DOI : https://doi.org/10.4000/ht.777

\section{(c) (†) $\ominus$}

La revue Horizons/Théâtre est mise à disposition selon les termes de la Licence Creative Commons Attribution - Pas d'Utilisation Commerciale - Pas de Modification 4.0 International. 


\section{ALINE JAULIN}

Aline Jaulin est doctorante à l'Université Paris Diderot - Paris 7, sous la direction d'Évelyne Grossman et d'Isabelle Barbéris, au sein de l'École doctorale 131, en histoire et sémiologie du texte et de l'image (Centre d'Études et de Recherches Interdisciplinaires en Lettres, Arts, Cinéma). Sa thèse s'intitule « Du médium aux dispositifs : théâtres contemporains de la médiation ». Elle a récemment publié "Exister à l'ère de la flexibilité chorégraphique : le cas des Gens d'Uterpan dans Avis d'audition », revue Agôn, dossier $n^{\circ} 7$ "L La Distribution ». Elle coordonne par ailleurs avec la géographe Élise Olmedo (Université Paris 1 - Géographie-Cités) le groupe de recherche «Écrire le sensible. Laboratoire itinérant de recherche-création » depuis mars 2015.

\section{Mail : a.alinejaulin@gmail.com}

Résumé: : Au XXle siècle, le ludisme infiltre la scène contemporaine, théâtrale, chorégraphique, performative. Les artistes s'inspirent de modèles de jeux préexistants - jeu de société, jeu de piste, jeu vidéo -, à l'instar du Jeu de l'oie du spectacle vivant de Julien Fournet (2010) et de Situation Rooms du collectif Rimini Protokoll (2013). S'inscrivant dans l'héritage de l'histoire de l'art depuis le début du $X X$ e siècle, imposant des règles, des canulars et des transgressions, ces formes artistiques placent le spectateur, devenu participant, au centre de la rela-

Abstract: In the twenty-first century $A D$, games begin to modify artistic creation in performing arts - theater, dance, living art. Artists draw on existing games, as board game, treasure hunt, video game, as Le Jeu de l'oie du spectacle vivant by Julien Fournet (2010) and Situation Rooms by Rimini Protokoll (2013). With their rules, hoaxes and transgressions, these artistic games have been part and parcel of art history since the early twentieth century. They are based on the audience as a participant. And sometimes, there is no performer anymore. These artistic games associate playful and critical tion théâtrale, sans que l'interprète y soit toujours nécessairement associé. Si elles concilient des exigences de divertissement et de fonction critique, ces formes tendent par ailleurs de plus en plus à s'autonomiser de leurs concepteurs. Nous verrons à travers les deux exemples analysés comment la scène contemporaine s'empare, par le jeu, de ces différents impératifs.

Mots-clés: scène contemporaine, jeu, théâtre, Julien Fournet, Rimini Protokoll

aims. At the same time though, they seem to be self-contained.

KEYwords: performing arts, game, theater, Julien Fournet, Rimini Protokoll 


\section{À quoi jouons-nous? Jouer, rejouer, déjouer la scène contemporaine}

$\ll$ On the seashore of endless worlds, children play. » Tagore

(«Sur le rivage de mondes sans fin, des enfants jouent ») in Jeu et réalité, l'espace potentiel, Winnicott, D. W., Paris, Gallimard, 1971 [1975, traduction française], p. 132.

La scène contemporaine - théâtrale, chorégraphique, performative interroge : « ¿A qué estamos jugando $?^{1}{ }^{1}$. Le titre du III Encuentro Creación Escénica Contemporánea ${ }^{2}$, tenu du 13 au 15 décembre 2012 à Valence en Espagne, met en évidence l'importance du jeu dans les réflexions qu'une partie du milieu théâtral mène actuellement. Le jeu, entendu ici en tant que principe ludique, c'est-à-dire non pas limité aux auteurs et comédiens mais déployé en dispositif autonome, place le spectateur au centre du processus. Ce déplacement, d'un «partage par le jeu » à un « partage $d u$ jeu $»^{3}$, infléchit profondément les fondements de la théâtralité ${ }^{4}$ jusqu'à, dans certains cas, la perdre de vue.

Bernard Dort, dans l'ouvrage La représentation émancipée, pointe déjà en 1988 la place du jeu dans les transformations à l'œuvre sur la scène théâtrale, du point de vue de l'espace, du comédien, du metteur en scène comme du spectateur. La dernière réflexion de cet essai s'attache à ce dernier : Bernard Dort observe combien la mise en jeu du spectateur vient bouleverser la conception traditionnelle de la représentation ${ }^{5}$. Cette mutation des pratiques théâtrales depuis les années 1980, mettant en avant le jeu au théâtre, s'impose aujourd'hui dans les pratiques et les discours. Il n'est pas seulement question de la part du jeu propre au théâtre, mais plus précisément de modalités de création reposant sur le modèle du jeu. Il existe de nombreux types de jeux inspirant la scène contemporaine : jeu vidéo, jeu de société ou jeu de piste entre autres. Citons par exemple les Jeux $W$ du collectif W (depuis 2003), les Jeux chorégraphiques de Laurent Pichaud et Rémy Héritier (2013), Go East, Jeu d'égarement de l'Agence Touriste (2014), Playmobile de la compagnie Divergences (2012), Insert Coin de T.R.A.N.S.I.T.S.C.A.P.E. (de Pierre 
Larauza et Emmanuelle Vincent, 2004) ou encore Le $23^{\text {ème }}$ joueur, ballon à comportements de Samuel Bianchini (2012). Héritiers d'une histoire de l'art considérée, selon les termes de Barbara Formis, comme un «immense champ d'expérimentation ludique $\gg^{6}$ depuis le début du $\mathrm{xx}^{\mathrm{e}}$ siècle, ces jeux théâtraux imposent leurs règles, leurs permis et leurs défendus, leurs canulars et leurs transgressions, travaillant depuis Winnicott les distinctions de game (jeu avec règles prédéfinies) et de play (jeu avec règles en train de se faire) ${ }^{7}$.

Nous proposons de nous intéresser ici à deux exemples de ce ludisme artistique : Situation Rooms du collectif allemand Rimini Protokoll (2013) et Le Jeu de l'oie du spectacle vivant de Julien Fournet, membre du collectif français l'Amicale de production (2010). Situation Rooms est construit comme un jeu vidéo à petite échelle et ne se présente que sous un seul format : un grand décor constitué de différentes pièces aménagées s'intègre à la scène des théâtres qui l'accueillent, activé par une vingtaine de spectateurs-participants à chaque session, sans autres spectateurs présents. Ce $\ll$ dispositif $^{8} \gg$ a déjà été programmé dans plusieurs continents et $s$ 'inscrit dans une scénographie monumentale et une dramaturgie constituée d'interviews filmées.

Le Jeu de l'oie du spectacle vivant se présente quant à lui sous deux formats différents : sous forme de jeu de société, avec un plateau dépliant disponible sur commande et facilement transportable, auquel quiconque peut décider de jouer en famille comme s'il s'agissait d'un jeu de l'oie traditionnel ; ainsi que sous forme d'installation et d'exposition, le jeu de l'oie étant alors programmé par les lieux culturels, exposé sur des murs et regardable comme une exposition.

Dans un cas comme dans l'autre, la première visée de ces propositions est de placer le spectateur-participant au cœur de la relation théâtrale, jusqu'à en faire disparaître l'artiste-concepteur et l'interprète. Cette tentation de fusion entre l'artiste et le public, oblitérant entre autres le comédien comme médiateur, réduit fortement la place de la théâtralité. Elle questionne le basculement à l'œuvre dans ces formes qui chercheraient moins, selon Christophe Triau, à « faire sens » qu'à « entretenir le lien, c'est-à-dire le jeu » entre les différents membres de la « communauté » artistique que le théâtre générerait ${ }^{9}$. Ainsi, en quoi le jeu, dans les formes artistiques contemporaines, renouvelle-t-il les questions de théâtralité ? Nous proposons en premier lieu de revenir sur l'importance historique du jeu dans l'histoire de l'art afin de mettre en évidence les valeurs de créativité et d'inventivité sur lesquelles celui-ci repose. Nous nous intéresserons ensuite aux effets du ludisme sur la théâtralité, ainsi qu'à l'idéal de communauté théâtrale qu'il cherche à convoquer. Nous 
nous demanderons enfin si la nécessité d'adaptabilité à laquelle ces formes répondent ne tendrait pas à annuler la dimension même de spectacle.

\section{«Partout comme aire de jeu ${ }^{10} »$ : la généralisation du jeu dans les formes artistiques}

De la plus tendre enfance à l'âge le plus avancé, on joue. Seul ou par équipes, parfois en réseau, on se plie à des systèmes de règles, de début, de fin, de permis et de défendu. La quantité de jeux disponible aujourd'hui est innombrable : jeux de société, jeux de hasard, jeux de plein air, jeux de piste, jeux de construction, jeux de rôles, jeux vidéo, etc. Selon Roger Caillois, la particularité du jeu, au-delà de l'atmosphère de délassement et de divertissement auquel il invite, est qu'il ne produit rien en apparence, ni biens, ni œuvres. En effet, même le principe des jeux de paris, où la dimension lucrative paraît essentielle, n'est qu'un déplacement des richesses d'un individu à un autre ${ }^{11}$. Cette gratuité apparente du jeu, malgré les limites imposées, est l'un des premiers éléments de définition couramment admis, associé à un imaginaire de liberté et d'invention. Pourtant, c'est également ce qui le discrédite le plus communément : derrière ses apparences d'aisance et de divertissement, il s'oppose encore souvent, dans l'imaginaire collectif, au modèle social du travail.

Or en 1971, Allan Kaprow, artiste et théoricien du happening, préconise dans la seconde partie de l'essai L'Éducation de l'Un-Artiste ${ }^{12}$ de substituer le jeu au travail, afin de permettre le déploiement d'une société plus créative. Dans cet essai, il conçoit le jeu comme une monnaie d'échange dans un monde où l'artiste n'a plus à tenir un rôle d'acteur mais à se faire « joueur ${ }^{13} \gg$. L'artiste permettrait ainsi aux individus de sortir de la course à l'industrialisation, de

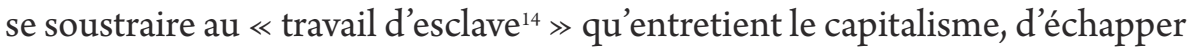
à l'esprit de compétition qui domine les relations humaines. Le jeu y est présenté comme inventif et instructif car il permet de susciter la participation du public.

Allan Kaprow s'inscrit plus largement dans une histoire de l'art du $\mathrm{Xx}^{\mathrm{e}}$ siècle qui, depuis les avant-gardes en particulier, fait du jeu une stratégie artistique de détournement des règles et des codes, dans l'héritage de ce que prônait Marcel Duchamp, celui d'un art envisagé comme un jeu entre tous les hommes de toutes les époques. De nombreux artistes contemporains ont ainsi fait du jeu le thème central de leurs œuvres, comme Ben avec Le jeu de la vie (1990) ou Maurizio Cattelan avec Stadium (1991). À partir de la fin des années 1990, de multiples expositions et événements thématiques rendent compte de cette émulation pour le jeu, en France et dans le monde : 
l'exposition Ecce Homo Ludens, le jeu comme art et mode de vie, réalisée dans le cadre de la manifestation régionale « Casanova Forever », à l'initiative de la Région Languedoc-Roussillon et pilotée par le FRAC L-R en 2010, ainsi que l'exposition Game Show, tenue en 2001 et en 2002 au Musée d'art contemporain du Massachussetts par exemple.

Dans le champ théâtral, Denis Guénoun soutient quant à lui qu' « il n'y a de spectateurs de théâtre, de notre temps, en notre monde, que comme joueurs en puissance ${ }^{15} \gg$. L'importance du jeu propre au théâtre, pleinement intégrée à la doxa contemporaine depuis les transformations à l'œuvre sur la scène artistique à la fin des années 1980 , se manifeste à la fois dans les déplacements des territoires théâtraux, où tout espace peut potentiellement devenir scénique, et dans la place accordée à la théâtralité. En effet, plus le jeu est au centre de la proposition artistique, plus la théâtralité apparaît infléchie, réduisant en même temps les dimensions réflexives et critiques traditionnellement au centre de la représentation théâtrale.

\section{Le ludisme et la fonction critique}

Dans Le Jeu de l'oie du spectacle vivant de Julien Fournet (2010) et Situation Rooms de Rimini Protokoll (2013), le jeu tend à dénoncer les paradoxes des logiques du marché capitaliste, qu'il s'agisse de ses conséquences dans la sphère du spectacle vivant ou plus largement dans les politiques économiques menées par les États au sujet de l'armement. La fonction critique communément attachée à la scène théâtrale semble ici, malgré le déplacement de la relation scène-salle vers un dispositif s'inspirant d'un modèle de jeu, contenue dans ces deux propositions. Mais dans ces exemples, il ne s'agit plus d'une fonction critique prise en charge par la représentation : en effet, le fait que le public participe, c'est-à-dire se fasse « joueur », produit un amoindrissement des distances entre l'œuvre et le spectateur. La fonction critique est ainsi déplacée dans la mesure où elle ne tient plus à distance scène et salle et qu'elle est mise en jeu par les dispositifs non-représentationnels. Cette réduction révèle par ailleurs un affaiblissement de la théâtralité : dans Situation Rooms, elle est maintenue par la scénographie monumentale et directive, alors que dans Le Jeu de l'oie du spectacle vivant, elle n'est tout simplement présente que dans les règles du jeu énoncées par Julien Fournet. On peut percevoir, entre ces deux exemples, un prisme de formes inspirées du jeu qui met à mal la théâtralité.

Le collectif Rimini Protokoll, composé de trois metteurs en scène allemands, Helgard Haug, Stefan Kaegi et Daniel Wetzel, propose depuis 2013 Situations Rooms, qui mêle installation, vidéo et performance et qui 
reprend l'idée d'un jeu vidéo mobilisant des spectateurs-participants ${ }^{16}$. Si le Théâtre des Amandiers, lors de sa diffusion en février 2015, le désigne comme un $\ll$ spectacle $^{17} \gg$, Rimini Protokoll, quant à lui, le caractérise comme « une pièce vidéo multijoueur ${ }^{18} \gg$, déplaçant les attentes traditionnellement associées au théâtre et au spectateur ${ }^{19}$. Le principe initial des concepteurs était de recréer un monde globalisé de la guerre et du commerce des armes à partir de vingt récits filmés de personnes réelles originaires de différents pays du monde ayant un lien direct avec l'armement (en tant que constructeur, trafiquant, combattant ou victime par exemple). Dans le théâtre, un décor monumental a été intégré à la scène, sorte de bâtiment composé de différents espaces posés à même le plateau, et vingt spectateurs-participants sont autorisés à y pénétrer à chaque session. Il s'agit de spectateurs lambdas qui sont invités par le personnel des théâtres à « activer » la proposition. Personne d'autre, en dehors de quelques membres du théâtre, n'assiste à cette «activation ». Chaque spectateur-participant est muni d'une tablette et d'un casque qui le guident, comme dans un jeu vidéo, pendant une heure trente, dans le dédale des pièces du décor, le faisant ainsi pénétrer dans la peau des vingt personnes interviewées au préalable. Il est invité non seulement à participer, mais également à agir au sein de la proposition artistique : déplacer des objets, ouvrir des portes, serrer les mains des autres spectateurs-participants par exemple. Ce jeu à l'échelle de la scène théâtrale cherche à poser un regard critique sur le système globalisé de l'armement, dans lequel chacun joue un rôle et se trouve motivé par des priorités politiques, économiques, sociales ou éthiques. Le dispositif monumental tend à réduire l'écart entre les acteurs et les spectateurs-participants, tout en cherchant à maintenir ce projet critique. Par la complexité de sa construction, associant déplacements dans les différents espaces et discours tenus par les interviewés auxquels assistent les spectateurs-participants, il est perceptible que Rimini Protokoll cherche à travailler constamment cette tension dialectique. Cela tient en particulier au maintien d'une construction dramatique : en dehors de la scénographie massive, chaque parcours de spectateur-participant repose surune dramaturgie précise, dans laquelle il doit intervenir physiquement. Ce spectateurparticipant est acteur du dispositif, en interaction avec les autres spectateursparticipants.

Dans Le jeu de l'oie du spectacle vivant ${ }^{20}$, et cela quel que soit le format (plateau de jeu familial ou affiches exposées dans des centres culturels), le principe est de proposer aux participants de traverser en quatorze étapes les turpitudes de la création, de la production et de la communication - de l'écriture du projet, étape 1, à la tournée, étape 14. Les règles sont clairement 
énoncées. Dans le plateau de jeu par exemple, elles se trouvent au centre. Elles précisent qu'il est nécessaire de former une équipe constituée d'un créateur/ trice, d'un chargé/e de production et d'un agent avant de lancer les dés et de suivre les indications. L'ensemble du jeu, satire du champ du spectacle vivant, en critique les logiques, pointant ses paradoxes et ses écueils. La première case du jeu souligne par exemple l'écart démesuré entre des velléités artistiques et les contraintes du milieu :

C'est une première création, mais vous avez choisi de travailler avec 17 interprètes, 1 ours, 1 piano à queue et 178 statuettes incas en carton que vous devez reconstruire tous les soirs.

Lancez le dé

PAIR - Vousfaites un compromis, vous serez 3 , et l'ours sera en peluche, relancez le dé.

IMPAIR - Redéfinition du projet, passez à la case suivante.

Julien Fournet s'approprie ainsi le modèle d'un jeu de société séculaire, le jeu de l'oie, pour dénoncer la complexité des stratégies artistiques à l'œuvre sur la scène contemporaine. Mais dans ce cas, si la thématique centrale est le spectacle vivant, dans la forme, la proposition ne relève pas de la représentation, seulement de l'expérimentation. Cela est particulièrement perceptible dans l'intitulé qui lui est donné, celui d'《Affiche/Installation ${ }^{21} »$. La forme intègre en elle-même la fonction critique attendue au théâtre, sous un angle discursif, sans passer par la médiation que serait la scène. La dimension critique n'est donc présente que dans les règles du jeu énoncées par Julien Fournet.

Les formes ludiques que nous avons évoquées en introduction, quelles qu'elles soient, déclinent, de façon plus ou moins distanciée, ces questions de théâtralité et de fonction critique, jusqu’à les dissoudre parfois complètement. De l'immersion du spectateur dans une forme dramatique inspirée du jeu au joueur détaché de toute dramaturgie et théâtralité, le participant voit remis en cause les espaces, éléments et fonctions dramatiques généralement dédiés au spectacle vivant. Face à ce déplacement manifeste des questions de représentation, c'est plus précisément la fonction de la relation théâtrale qui est transformée.

\section{Rejouer et déjouer la communauté théâtrale}

Vincent Romagny, en introduction à l'ouvrage collectif Anthologie des aires de jeux d'artistes, défend l'hypothèse selon laquelle les artistes, créant à partir du jeu, « pensent et travaillent les conditions du "vivre ensemble”22 $\gg$. Par leur 
capacité à réunir dans un même lieu des individus différents durant un temps circonscrit, qu'il s'agisse du théâtre, de la friche, du musée, de la ville ou d'un espace naturel, ils créeraient les conditions de l'expérience sensible, d'une mise en relation avec soi, avec l'autre et avec l'environnement, d'une mise en commun de pratiques et de valeurs. Les psychanalystes ont reconnu l'importance décisive du jeu dans l'histoire de l'affirmation de soi chez l'enfant et plus largement dans le développement de l'individu et des sociétés. Pour Winnicott par exemple, « jouer conduit à établir des relations de groupe ${ }^{23}$ », c'est seulement en jouant que l'individu part à la quête du soi, qu'il peut se rassembler et exister en tant qu'unité, dans l'expression de sa créativité. Cette condition de l'expérience concerne l'individu mais également les relations qu'il entretient avec son entourage, et que Winnicott observe dans la relation psychothérapeutique.

Le théâtre se trouve en ce sens au centre des discussions théoriques, dans la mesure où il apparaît comme un rempart à ce délitement d'une communauté « désœuvrée ${ }^{24}$ », communauté au sens donné par George Yudice, soit « un ensemble existant de relations, impliquant une connexion - comme la parenté, l'héritage culturel, les valeurs et les buts partagés $[\ldots]^{25} \gg$. Le théâtre est le lieu où on continue aujourd' hui à se poser la question : « comment produire de la communauté, cette communauté étant toujours une fiction ? », ce qui interroge par exemple Marie-José Mondzain ${ }^{26}$. Il est perceptible que Situation Rooms travaille cette question à travers son dispositif monumental. En effet, chacun des participants, muni de sa tablette et de son casque, est amené à suivre une ligne dramatique propre. Dans ce cadre, il reçoit des informations de l'extérieur (entretiens filmés et indications au casque) et est appelé à agir sur le déroulement du dispositif. Dans ce monde extérieur, qui associe des informations issues des nouvelles technologies de l'information et de la communication (NTIC) et des informations issues de la scénographie, il entre en contact, à distance ou physiquement, avec les autres participants. Différentes interactions sont proposées par le dispositif : se croiser dans les salles et les couloirs, serrer la main d'un autre participant, lui donner un accessoire par exemple. Le point d'orgue de cette mise en jeu d'une « communauté théâtrale $\gg$ advient lorsque tous les participants sont réunis dans la plus grande pièce du dispositif, la salle de conférences, à la fin. En effet, même si certains se sont croisés ou sont entrés en contact auparavant, c'est à ce moment-là que les vingt spectateurs-participants présents se retrouvent ensemble, immobiles, casques et écrans en main, se voyant et s'observant tous pour la première fois, dans une proximité physique et une disposition spatiale circulaire privilégiant la rencontre des regards. Cette configuration, si 
elle interroge la notion de communauté, la place du point de vue du réseau : ce n'est plus une communauté physiquement unie dans un même lieu, la salle de spectacle, conviée à partager la même représentation, mais bien vingt expériences de spectateurs-participants, élaborées sur le modèle du réseau, qui sont ici privilégiées. La réunion finale en une seule et même « communauté théâtrale » pose pourtant question : suffit-il d'avoir été réunis quelques instants dans une même pièce, après avoir partagé des parcours similaires, mais néanmoins distincts, pour « faire communauté » ? Les applaudissements gênés, puis la dispersion rapide des participants à la fin de Situation Rooms suggèrent l'inverse. Le sujet, pour Rimini Protokoll, est peut-être moins de tenter de composer une communauté idéale que d'en souligner les limites. Il n'en demeure pas moins que cette fin, aussi cohérente soit-elle d'un point de vue dramatique, suscite de la gêne, perceptible dans les regards et expressions des participants.

Dans le cas du Jeu de l'oie du spectacle vivant, la question de la communauté théâtrale apparaît moins jouée que déjouée dans la mesure où elle est principalement convoquée par des détournements comiques. Certaines cases du jeu de Julien Fournet, en particulier « Étape 11 - Première », mettent en lumière les aléas de la représentation et de la versatilité du public :

Une série de problèmes techniques ruine la première, heureusement ce soir c'est la finale de l'euro et personne n'est venu.

Avancez étape 12

Une série de problèmes techniques ruine la première, malheureusement, ce soir c'est la finale de hockey sur gazon et tout le monde est venu.

Retour étape 10

Ces énoncés, en apparence anodins, montrent la complexité à l'œuvre dans l'idée de constitution d'une communauté théâtrale. La présence des spectateurs est soumise aux variations de l'actualité sportive et de sa diffusion massive sur les écrans. Cette opposition révèle la fragilité des arts de la présence face à des divertissements relayés par les médias. En réalité, en proposant non pas une forme représentationnelle mais un double format plateau de jeu familial et affiche/exposition, Julien Fournet ne met que plus férocement en évidence la faillite de la notion d'assemblée théâtrale : pas de spectacle, pas de communauté, même si elle est présente dans les règles du jeu énoncées. C'est l'espace même de l'assemblée théâtrale qui est en péril lorsque le lieu de la scène n'est plus privilégié, comme le souligne Chris Balme : « dans un monde où l'esthétique est dispersée ou distribuée, il est difficile de réussir à localiser le théâtre ${ }^{27} \gg$. 


\section{Jouer le jeu de l'adaptabilité}

Depuis la fin du $\mathrm{Xx}^{\mathrm{e}}$ siècle, en particulier dans le monde occidental, nous sommes entrés dans l'ère du ludisme globalisé : les jeux vidéo par exemple, jeux électroniques impliquant une interaction humaine avec une interface utilisateur, lorsqu' ils sont mis en réseau, permettent de jouer, depuis chez soi, avec n'importe qui d'autre dans le monde disposant du même accès au réseau. La scène contemporaine s'est saisie de ce nouveau régime du ludisme, concevant des formes modulables et exportables aisément, appelant les artistes à produire des formes adaptées aux contextes, aux publics, ainsi qu'aux contraintes budgétaires associées. Rimini Protokoll, qui a déjà présenté le projet inspiré des jeux vidéo Situation Rooms sur plusieurs continents, évoque ainsi sa mise en scène de la globalisation :

Situation Rooms' gathers together from various continents 20 people whose biographies have been shaped by weapons in a film set that recreates the globalised world of pistols and rocket-propelled grenades, of assault rifles and drones, of rulers and refugees, becoming a parcours of unexpected neighbourhoods and intersections ${ }^{28}$.

Si Situation Rooms cherche à rendre manifeste les implacables logiques de communication et d'interaction que l'époque contemporaine provoque, il questionne et fragilise en même temps le fonctionnement et l'économie du spectacle vivant. En effet, avec ce type de dispositif, il n'y a par exemple plus de nécessité à financer des interprètes : autonomes des artistes qui les conçoivent, ces formes ne nécessitent pas obligatoirement la présence d'acteurs, voire d'artistes, pour exister au-delà de leur conception. Cet aspect n'est pas spécifique aux propositions reposant sur des modèles de jeux, mais traverse plus largement la scène contemporaine, comme l'attestent par exemple Le Sacre du printemps de Roméo Castellucci (ballet mécanique élaboré à partir de poussière d'os diffusée par une complexe machinerie, 2014) ou la pièce $E l$ Triunfo de la Libertad de La Ribot, Juan Dominguez et Juan Loriente (centrée sur un texte transmis par une interface sans aucun comédien en scène, 2014).

Dans Situation Rooms, les vingt spectateurs-participants sont accueillis dans une salle vide. Seuls quelques membres du personnel du théâtre sont présents, les invitant à se délester de leurs affaires et à se munir du matériel mis à disposition. Ensuite, poussé à jouer différents rôles, de la victime au bourreau, chacun croise d'autres spectateurs-participants jouant eux-mêmes des figures de l'industrie de l'armement, jusqu'à la scène finale, réunissant les vingt personnes dans le même espace. Ces rencontres ne font que renforcer 
le sentiment d'absurdité qui se dégage d'un système pour lequel les questions éthiques sont subordonnées aux priorités économiques. Les faibles applaudissements - il n'y a alors personne, ni interprète, ni concepteur, seulement deux ou trois membres du théâtre pour les recevoir -, puis la dispersion rapide des spectateurs-participants, sans savoir qui saluer, mettent en évidence cette absence.

De la même façon, Le jeu de l'oie du spectacle vivant, en format installation/exposition et en format affichette/plateau de jeu dépliant, apparaît autonomisé de ses concepteurs, sans interprète nécessaire à sa réalisation, objet diffusable à loisir sans impliquer de relation humaine. Pour le format installation/exposition, l'Amicale de production précise pourtant que « des conférences ou des ateliers peuvent également être menés par Julien Fournet autour du projet $[\ldots]^{29} \gg$. L'usage du verbe « pouvoir » marque en réalité le caractère facultatif de l'intervention humaine, pour laquelle l'artiste est ici présenté comme un médiateur optionnel. Dans la même logique, l'invitation à se procurer le jeu format affichette/plateau de jeu en le commandant sur le site de la scène nationale de Valenciennes, le Phoenix, révèle cette vacance de la rencontre humaine dans le système de production artistique, de la création à la réception.

« Quatorze euros quatre-vingt-dix »: sur le site du Phoenix, c'est le prix du Jeu de l'oie du spectacle vivant, intégré à un coffret intitulé Exhibition des rouages et logistique de la monstration. Prix similaire à n'importe quel jeu de société dans une grande surface, Le jeu de l'oie du spectacle vivant, sous-intitulé «Un jeu de rythme et de justesse. Une histoire d'échelle et de moyens », reconduit ainsi ce qu'il cherche par ailleurs à dénoncer, une économie de la précarité dans laquelle l'artiste, afin d'exister dans le champ du spectacle vivant, cherche à «se vendre », quel qu'en soit le prix. Dans la vignette «Étape 13, diffusion », Julien Fournet l'exprime par exemple ainsi :

Vous avez très bien vendu le spectacle (un montage de textes de Cioran) à un fest-noz : les 3 comédiens sont outrés, ils quittent le projet.

Retournez étape 7

Tournant en dérision la problématique de la nécessité, pour un artiste, de s'adapter à son contexte, Julien Fournet souligne en même temps la difficulté d'exister dans le milieu du spectacle vivant sans abandonner des priorités esthétiques et éthiques. 
Alors, à quoi jouons-nous ? La scène contemporaine, traversée par le modèle du jeu, cherche à concilier des exigences de divertissement, de fonction critique mais aussi d'idéal de constitution d'une « communauté théâtrale ». Elle est en réalité à saisir dans l'« économie de la précarité » soulevée par le III Encuentro Creación Escénica Contemporánea, dont le sous-titre de l'événement ¿A qué estamos jugando? est : « Comunidad, juego y economías de la creatividad precariedad », le terme « creatividad » ayant été volontairement barré30. Les formes artistiques du jeu, qui intègrent la participation du public, permettent alors de réduire le financement d'interprètes, d'autonomiser l'œuvre par rapport à ses concepteurs et de l'exporter facilement. Dans cette tension entre différentes injonctions émergent ainsi des modèles de jeux que la scène contemporaine tend à favoriser, aux dépens des interprètes, de la théâtralité, mais aussi de l'artiste qui, pour Bernard Dort, « perd de sa suzeraineté ${ }^{31} »$.

\section{Notes}

1. «À quoi jouons-nous? ? (notre traduction).

2. ¿̇A qué estamos jugando? Comunidad, juego y economías de la creatividad precariedad (mise en forme originale), III Encuentro Creación Escénica Contemporánea, du 13 au 15 décembre 2012, Valence, Espagne.

3. Christophe Triau, «Lillusion ludique » in Théâtre/Public «Vers une nouvelle séquence théâtrale européenne ?» n 194, sept. 2009, p. 37.

4. En écho à la définition de Bernard Dort, La représentation émancipée, Arles, Actes Sud, 1988, p. 183 : «La théâtralité, alors, n'est plus seulement cette "épaisseur de signes" dont parlait Roland Barthes. Elle est aussi le déplacement de ces signes, leur impossible conjonction, leur confrontation sous le regard du spectateur de cette représentation émancipée. »

5. Ibid.

6. Barbara Formis, "L'art en jeu » in Vincent Romagny (sous la dir. de), Anthologie des aires de jeux d'artistes, Quimper, Editions Infolio, Le Quartier, 2010, p. 117.

7. Donald Woods Winnicott, Jeu et réalité, l'espace potentiel, Paris, Gallimard, 1971 [1975, traduction française].

8. Arnaud Rykner, «Du dispositif et de son usage au théâtre », in Tangence n ${ }^{\circ} 88$ : Devenir de l'esthétique théâtrale, sous la direction de Gilbert David et Hélène Jacques, Presses de l'université du Québec, automne 2008.

9. Christophe Triau, op. cit., p. 37.

10. Allan Kaprow, «L'éducation de l'Un-Artiste, deuxième partie » [1972], L'art et la vie confondus, textes réunis par Jeff Kelley, traduction par Jacques Donguy, Paris, Éditions du Centre Pompidou, 1996, p. 145.

11. Roger Caillois, Les jeux et les hommes [1958], Paris, Gallimard, 1967, p. 10.

12. Allan Kaprow, op. cit. 
13. Ibid., p. 159.

14. Ibid., p. 145.

15. Denis Guégoun,Le théâtre est-ilnécessaire?, Belval, Circé,1997,p. 165-66 in Christophe Triau, op. cit., p. 36.

16. Collectif Rimini Protokoll, Site de Rimini Protokoll. [En ligne]. Rimini Protokoll [Page consultée le 10 avril 2016]. Disponibilité et accès http://www.rimini-protokoll. de/website/en/project_6009.html. Situation Rooms présenté à plusieurs reprises en France : Parc de la Villette en 2013, Théâtre Nanterre-Amandiers en 2015.

17. Théâtre Nanterre-Amandiers, Site de Théâtre Nanterre-Amandiers. [En ligne]. Amicale de production [Page consultée le 15 septembre 2016]. Disponibilité et accès http://www.nanterre-amandiers.com/2014-2015/situation-rooms/.

18. "A multiplayer video piece ", Site de Rimini Protokoll, op. cit.

19. En ce qui concerne la place du spectateur, voir : Anyssa Kapelusz, « De la "participation” au "participatif" : évolution de la place du spectateur ", Jeu : revue de théâtre, n 147 (2), 2013, p. 58-63.

20. Amicale de production, Site de l'Amicale de production. [En ligne]. Amicale de production [Page consultée le 10 avril 2016]. Disponibilité et accès http://www.amicaledeproduction.com/projets/jeudeloie.php.

21. Ibid.

22. Vincent Romagny (dir.), Anthologie des aires de jeux d'artistes, Quimper, Éditions Infolio, Le Quartier, 2010, p. 7.

23. Donald Woods Winnicott, Jeu et réalité, l'espace potentiel, op. cit., p. 60.

24. Jean-Luc Nancy, La communauté désceuvrée, Paris, Bourgeois, 1986.

25. George Yudice, « Community », New Keywords, Blackwell, 2005, p. 51 cité par Patrice Pavis, Dictionnaire de la performance et du théâtre contemporain, Paris, Armand Colin, 2014, p. 48.

26. Marie-José Mondzain, L'assemblée théâtrale, Paris, Éditions de l'Amandier, 2002, p. 129.

27. Chris Balme, «Distributed aesthetics ", Theatrical Blends, Jerzy Limon et Agnieszka Zukowska (ed.), 2010, p. 146, cité par Patrice Pavis, Dictionnaire de la performance et du théâtre contemporain, Paris, Armand Colin, p. 49.

28. «Situation Rooms réunit vingt personnes issues de différents continents, dont les biographies ont été façonnées par le commerce des armes dans un ensemble de films qui recrée les échanges à l'échelle mondiale de pistolets et de grenades auto-propulsées, de fusils d'assauts et de drones, de dirigeants et de réfugiés, devenant un parcours de voisinages et d'intersections inattendus " (notre traduction).

29. Site de l'Amicale de production, op. cit.

30. "Communauté, jeu et économie de la eréativité de la précarité » (notre traduction).

31. Bernard Dort, op. cit., p. 183. 